\title{
Хроническая обструктивная болезнь легких: особенности у женщин
}

ГОУ ВПО "ММА им. И.М.Сеченова": 119991, Москва, ул. Трубецкая, 8, стр. 2

\section{S.I.Ovcharenko, V.A.Kapustina \\ Chronic obstructive pulmonary disease: particularities in women}

Key words: chronic obstructive pulmonary disease, gender differences, susceptibility, women.

Ключевые слова: хроническая обструктивная болезнь легких, гендерные различия, предрасположенность, женщины.

Хроническая обструктивная болезнь легких (ХОБЛ) является одним из наиболее распространенных заболеваний и одной из ведущих причин смерти во всем мире [1]. Как известно, ХОБЛ - это хроническое воспалительное заболевание, возникающее под воздействием различных факторов риска, главным из которых является табакокурение, и протекающее с преимущественным поражением дистальных отделов дыхательных путей и паренхимы легких и с формированием эмфиземы. Она характеризуется частично обратимым или, с течением времени, необратимым ограничением скорости воздушного потока, индуцированным воспалительной реакцией, и проявляется кашлем, продукцией мокроты и нарастающей одышкой. ХОБЛ развивается у предрасположенных лиц и отличается неуклонным прогрессированием, в результате чего развивается хроническая дыхательная недостаточность [2].

Хотя заболевание в первую очередь поражает легкие, для него характерны и значимые системные проявления [3]. В их основе лежат разнообразные механизмы, пока недостаточно изученные. Среди причин развития системных проявлений, помимо гипоксемии, курения, малоподвижного образа жизни, важное место занимает системное воспаление, вовлекающее в процесс различные органы и системы. К основным внелегочным проявлениям ХОБЛ относятся снижение питательного статуса, дисфункция скелетных мышц, остеопороз, анемия, сердечно-сосудистые эффекты, а также нервно-психические расстройства, проявляющиеся нарушениями памяти и сна, депрессией, возникновением страхов.

До недавнего времени большинство врачей типичным пациентом с ХОБЛ считали пожилого мужчину, страдающего кашлем и одышкой после долгих лет курения, регулярно переносящего сезонные обострения бронхита. Однако клиническая практика последних лет показывает, что контингент пополнили курящие женщины среднего возраста, а в ряде стран, таких как Китай, Индонезия, Южная Азия, и некурящие женщины, контактирующие с органи- ческим топливом и продуктами его сгорания. В соответствии со сложившимся стереотипом, когда на прием к терапевту или пульмонологу приходит курящий мужчина с респираторными симптомами, в первую очередь возникает мысль о ХОБЛ, в то время как при консультации женщины со значительным стажем курения и жалобами на состояние органов дыхания наличие этого заболевания, к сожалению, подозревают не сразу.

Как и исследователи сердечно-сосудистой патологии, научное сообщество, занимающееся изучением болезней органов дыхания, в последнее время все больше внимания уделяет влиянии пола пациента на диагностику и лечение ХОБЛ. Используемый в иностранной медицинской литературе термин "род" (gen$d e r)$ и применяемый в отечественной - "пол" (sex) не являются тождественными. Понятие "пол" применимо к первичным и вторичным половым признакам мужчин и женщин, но часто используется, чтобы охватить все анатомические и физиологические особенности, которые могут у них различаться. Род представляет собой результат социальных, экологических, культурных и физиологических признаков, которые можно назвать мужскими ("маскулинными") и женскими ("фемининными") и которые позволяют человеку идентифицировать себя как мужчину или женщину [4]. В дальнейшем речь пойдет о гендерных различиях, хотя надо понимать, что в основе многих из них могут лежать генетические и биологические особенности рода человека.

\section{Эпидемиология ХОБЛ и гендерные различия}

Распространенность, заболеваемость и смертность от ХОБЛ во всех странах недооцениваются. Хотя распространенность является одним из основных показателей эпидемиологии, проблемы ХОБЛ в этой области решены далеко не полностью. В нашей стране, по усредненным данным Минздравсоцразвития РФ, распространенность ХОБЛ в 2002 г. составила 1610,8 на 100000 населения $(1,6 \%)$ [5]. При этом 
размах колебаний показателей очень велик: от 996,5 на 100000 населения ( 1 \%) до 6 249,4 на 100000 населения $(6,2 \%)$, что, скорее всего, свидетельствует не об истинных различиях в распространенности заболевания, а об уровне его диагностики в разных регионах страны. Трудность интерпретации этих показателей в значительной степени связана с недостаточным проведением в России современных многоцентровых стандартизованных эпидемиологических исследований.

Исторически распространенность ХОБЛ была значительно выше среди мужчин, по сравнению с женщинами. Это можно объяснить различной экспозицией факторов риска, в частности табакокурения. Вместе с тем в настоящее время отмечается рост заболеваемости среди женщин, у которого есть несколько причин.

По данным исследований, проведенных в США, с 1982 по 1996 гг. заболеваемость хроническим бронхитом возросла как среди мужчин, так и среди женщин. Среди женщин распространенность увеличилась на $65 \%$, а за тот же период среди мужчин только на $50 \%$ [6]. Сходная эпидемиологическая ситуация сложилась в Великобритании. С 1990 по 1997 гг. распространенность ХОБЛ возросла на $25 \%$ среди мужчин и на $69 \%$ среди женщин [7]. Каждый год количество новых случаев ХОБЛ среди лиц женского пола растет приблизительно в 3 раза быстрее, чем среди мужчин. По прогнозам специалистов распространенность ХОБЛ за 20 лет (с 1994 к 2015 гг.) среди мужчин увеличится на $43 \%$, а среди женщин на $142 \%$ [8].

Помимо широкой распространенности чрезвычайно важной характеристикой ХОБЛ является ее неуклонно прогрессирующее и инвалидизирующее течение, что приносит социально-экономический ущерб не только самим больным, членам их семей, но и обществу в целом. Бремя государственных экономических затрат, связанных с ХОБЛ, очень велико. Так, например, только в США в 2002 г. расходы, обусловленные этим заболеванием, составили 32,1 млрд долларов [9].

Другим важным эпидемиологическим показателем, который неуклонно продолжает расти, когда речь идет о ХОБЛ, является смертность. Занимая в начале нашего столетия 4-5-е место среди причин смертности в возрастной группе старше 45 лет и унося жизни около 3 млн человек в год, ХОБЛ остается единственным заболеванием, уровень летальности вследствие которого не только не снижается, но продолжает увеличиваться. По неутешительному прогнозу экспертов Всемирной организации здравоохранения (ВО3) к 2020 г. ХОБЛ войдет в 1-ю тройку заболеваний, лидирующих по показателям летальности, обусловливая около 4,7 млн смертей в год [1, 2].

По данным ВО3, летальность при ХОБЛ в РФ составляет 16,2 на 100000 населения, что сопоставимо с большинством европейских стран. Вместе с тем, согласно официальной статистике Минздравсоцразвития РФ, диапазон колебаний показателя смертности при хронических болезнях нижних дыхатель- ных путей весьма значителен - от 13,1 в Дальневосточном федеральном округе до 86,6 в Курской обл. на 100000 населения [5].

В наблюдении, охватывающем период с 1970 по 2002 гг, среди жителей США отмечается рост смертности от ХОБЛ, в то время как показатели летальности от других основных причин снижаются [10]. Как указывают K.M.Brett и S.G.Hayes [11] в настоящее время ХОБЛ вызывает большинство смертей среди лиц женского пола. По статистике, во всем мире от ХОБЛ умирает женщин больше, чем от рака молочной железы и рака легких вместе взятых [12]. В Канаде рост летальности вследствие ХОБЛ среди женщин наиболее заметен после 1990 г.; при этом прогнозируется, что в ближайшие годы этот показатель среди женщин будет увеличиваться значительно быстрее, чем среди мужчин [13]. В США рост летальности от ХОБЛ среди женщин зафиксирован после 1970 г., и к 2000 г. уровень смертности от данного заболевания в стране был выше среди женщин, чем среди мужчин [14]. По данным J.Vestbo et al., за 10 лет летальность среди мужчин увеличилась на $17,1 \%$ (с 96,3 до 112,8 на 100000 человек), а среди женщин - на 126,1 \% (с 24,5 до 55,4 на 100000 человек) [15].

По прогнозам многих мировых исследователей уровень смертности от ХОБЛ среди женщин в ближайшее время будет продолжать расти и опередит показатели среди мужчин.

\section{Факторы риска ХОБЛ и гендерные различия}

Табакокурение - главный этиологический фактор развития ХОБЛ. В мире насчитывается порядка 1,3 млрд ежедневно курящих людей. Если потребление табака останется прежним, их количество к 2025 г. возрастет до 1,7 млрд [16], а около $1 / 2$ из них, возможно, умрут от заболевания, ассоциированного с табакокурением [17]. По статистике, число курящих среди мужчин незначительно снижается, однако среди женщин - существенно возрастает. Как ожидается, к 2025 г. > 500 млн женщин будут курить, что составит порядка $20 \%$ женского населения планеты (по сравнению с нынешними $12 \%$ ) [18, 19].

Эпидемиологические исследования показали, что распространенность табакокурения среди населения Российской Федерации достаточно высока. Количество курящих женщин в течение многих лет было небольшим, но значительный его рост стал отмечаться в последние 15 лет. В середине 80 -х гг. ХХ в. распространенность табакокурения составляла 3-5\%, а в начале XXI в. - уже 20-30\%. В различных регионах число курящих женщин колеблется от 10 до $50 \%$. Каждая 3-я женщина в возрасте 18-19 лет курит [20]. При этом активность курения изменяется в зависимости от социально-экономического статуса. Эпидемиологическое исследование, проведенное среди жителей Москвы, показало, что у работающих женщин с высшим образованием его распространенность составляет $33,7 \%$, в то время как среди женщин без специального образования - 50 \% [21]. 
Данные о высокой предрасположенности женщин к курению неоднозначны. Так, большинство исследователей считают, что женщины более подвержены развитию ХОБЛ вследствие особенностей метаболизма табачного дыма. В основе могут лежать несколько биологических механизмов: увеличение осаждения токсических веществ в легочной ткани у женщин, ухудшение клиренса и усиление ответной реакции на токсические вешества. Сила ответной реакции может быть обусловлена трансформацией ксенобиотиков в более токсичные метаболиты либо снижением их конъюгации и экскреции. Показано, что у курящих женщин, в отличие от мужчин, в легочной ткани экспрессируется большее количество цитохрома Р450, являющегося медиатором в окислении ксенобиотиков [22]. Известно, что регуляторами экспрессии и активности цитохрома Р450 являются половые гормоны. Так, стимуляция эстрогеновых рецепторов в легочной ткани приводит к усилению выработки этого фермента и метаболизма сигаретного дыма, в ходе которого синтезируются оксиданты. В связи с тем, что эстрогены не влияют на экспрессию и активность ферментов, принимающих участие в детоксикации ксенобиотиков (глутатион), оксидативный стресс, приводящий к повреждениям легочной ткани, больше выражен у курящих женщин, чем у мужчин [23]. Этим гормональным воздействием можно объяснить усиленный метаболизм никотина и других веществ, содержащихся в табачном дыме, у женщин [24].

В Пекинском респираторном исследовании здоровья [25], в котором участвовали 1618 мужчин и 1669 женщин в возрасте 40-69 лет, процент курящих был выше среди мужчин (78 \% vs $35 \%$ ). Несмотря на то, что основные показатели легочной функции были лучше у никогда не куривших женщин по сравнению с никогда не курившими мужчинами, эти же показатели оказались хуже у курящих женщин, нежели у курящих мужчин. Исследователи предполагают, что неблагоприятное влияние табачного дыма на легочную функцию более выражено у лиц женского пола, но не является следствием гендерных различий в распространенности курения. В другом исследовании, проведенном в Китае и посвященном падению объема форсированного выдоха за 1-ю с $\left(\mathrm{OФB}_{1}\right)$ на фоне курения в зависимости от пола больного, в котором принимали участие $>4500$ человек, наблюдавшихся в течение 24 лет, показано более значимое негативное воздействие табакокурения на легочную функцию у женщин [26].

E.Prescott et al. [27] сравнили результаты 2 крупных европейских поперечных исследований, посвященных курению и снижению легочной функции. В Копенгагенском городском исследовании сердца CCHS, включавшем 13897 человек, и Национальных исследованиях Глострап (GPS) с участием 4816 пациентов уровень избыточного падения легочной функции из расчета на каждую выкуренную за год пачку сигарет составил, по данным CCHS, 7,4 мл у женщин и 6,3 мл у мужчин. По результатам GPS, тот же показатель оказался несколько выше, но падение легочной функции происходило интенсивнее у женщин, чем у мужчин: 10,5 мл и 8,4 мл соответственно.

R.Carter et al. [28] в своем исследовании пришли к схожим выводам. При одном и том же уровне потребления табака степень развивающейся бронхиальной обструкции (тяжесть ХОБЛ) была всегда выше у женщин по сравнению с мужчинами.

В то же время не все исследователи разделяют мнение о большей предрасположенности женщин к развитию бронхообструкции, полагая, что мужской пол сам по себе является фактором риска ХОБЛ. В 3 продольных исследованиях, проведенных среди курильщиков в США [29], Австралии [30], а также в рамках Исследования здоровья легких LHS [31] не доказано столь же негативное воздействие табачного дыма на показатели легочной функции у женщин, как у мужчин. По результатам исследования, проведенного в Испании [32], из 795 курильщиков у $26 \%$ выявлена ХОБЛ (30,5\% мужчин и 22,3\% женщин со схожей степенью бронхообструкции). У лиц старше 50 лет заболевание в 2 раза чаще встречалось среди мужчин (34 \% vs 17 \%). При анализе частоты заболевания в зависимости от стажа и интенсивности курения распространенность ХОБЛ оказалась ниже среди женщин, что позволяет судить об их меньшей предрасположенности к развитию бронхиальной обструкции.

Согласно эпидемиологическим данным, среди пациентов с ХОБЛ не курят всего 5-12 \%. Группа ученых из Великобритании [33] попыталась выявить клинические, рентгенологические и патофизиологические особенности заболевания среди пациентов без анамнеза курения либо с индексом курения < 5 пачек / лет. В изучаемой когорте преобладали женщины (86 \%). Особенности, выявленные при компьютерной томографии высокого разрешения, оказались неспецифическими и были присущи всем больным ХОБЛ. Существенными оказались изменения, обнаруженные в ходе цитологического анализа мокроты, а именно увеличение количества нейтрофилов и эозинофилов, что позволило разделить обследуемых на 2 подгруппы. Так, в подгруппе больных ХОБЛ с повышенным содержанием нейтрофилов в мокроте чаще встречались аутоиммунные заболевания, в частности аутоиммунный тиреоидит. Значимость такого наблюдения пока сложно оценить, однако убедительным кажется тот факт, что некоторые случаи ХОБЛ, особенно у некурящих, развиваются в результате нарушения иммунной регуляции, которое чаще происходит у женщин.

Хотя тенденция к увеличению потребления табака является одной из основных причин роста заболеваемости ХОБЛ среди женщин, нельзя исключать влияние других факторов риска. Например, в Китае курят < $10 \%$ женщин, однако в 2000 г. в этой стране ХОБЛ стала 2-й среди ведущих причин смертности у женщин старше 65 лет [34]. Наряду с табакокурением не менее важную роль в заболеваемости ХОБЛ, особенно в развивающихся странах, играет состояние окружающей среды [35]. По данным исследований, проведенных в Индии [36], Китае [37], Непале [38], 
Иране [39], Саудовской Аравии [40] и Мексике [41], загрязнение воздуха продуктами сгорания биоорганического топлива при приготовлении пищи в плохо вентилируемых помещениях приводит к повышению распространенности хронических респираторных заболеваний у женщин и ежегодно служит причиной смерти 2 млн женщин и детей. Очевидно, что, какими бы ни были причины развития обструктивных нарушений, женщины находятся в группе повышенного риска [42].

Заслуживают внимания данные о роли гиперреактивности бронхов в формировании ХОБЛ. Известно, что гиперреактивность бронхов ассоциирована с высоким риском прогрессирования и смертности от ХОБЛ. Приблизительно у $87 \%$ курящих женщин с ХОБЛ легкой и средней степени тяжести выявляется высокая бронхиальная обратимость, тогда как у курящих мужчин - лишь в $63 \%$ случаев [43]. У мужчин основными факторами риска развития гиперреактивности бронхов являются атопия и бронхиальная астма (БА), в то время как у женщин единственный наиболее значимый фактор риска - табакокурение [44].

Накопленные данные позволяют говорить о более выраженной обратимости бронхиальных нарушений у лиц женского пола. Предполагают, что высокая гиперреактивность бронхов у женщин может быть связана с исходно меньшим калибром бронхиального дерева, по сравнению с мужчинами [45, 46]. При меньшем просвете бронхов ожидается большее снижение ОФВ 1 во время бронхопровокационного теста, поскольку сокращение кольцевых гладкомышечных волокон бронхов ведет к более сильному нарастанию резистентности в узких просветах бронхов, по сравнению с широкими.

Исходное значение ОФВ 1 часто используют для оценки просвета бронхов. Но в связи с тем, что значение этого показателя у мужчин физиологически выше, сравнение мужчин и женщин со сходным уровнем ОФВ 1 эквивалентно сопоставлению мужчин с высоким риском гиперреактивности бронхов с женщинами, у которых риск гиперреактивности бронхов невысок.

Другим гипотетическим объяснением гендерных различий в предрасположенности к респираторным заболеваниям может быть особый гормональный фон женщин, хотя его влияние до сих пор дискутируется. Полагают, что эстрадиол влияет на концентрацию ацетилхолина, характер секретируемой бронхиальной слизи, продукцию простагландинов и плотность $\beta_{1}$ - и $\beta_{2}$-адренергических рецепторов в легочной ткани [47]. В течение репродуктивного периода у женщин происходят циклические изменения в респираторном тракте, которые проявляются большей распространенностью гиперреактивности бронхов по сравнению с мужчинами.

Более того, в связи с циклической гормональной активностью у женщин исходно определяется более высокая концентрация провоспалительного интерлейкина-8 (IL-8), по сравнению с мужчинами [48]. Данный цитокин рассматривается в качестве специфичного биологического маркера ХОБЛ и является хемоаттрактантом для нейтрофилов, привлекая их в легочную ткань. Курение способствует нарастанию уровня IL-8, что приводит к еще большему увеличению содержания нейтрофилов в дыхательных путях [49]. Поэтому лица женского пола особенно чувствительны к патологическому воздействию табачного дыма [50].

Сами по себе внешние факторы, такие как загрязнение окружающей среды, не вызывают развития ХОБЛ. Их влияние реализуется при наличии генетической предрасположенности. Помимо наиболее известного эндогенного фактора риска, предрасполагающего к развитию эмфиземы, - дефицита фермента $\alpha_{1}$-антитрипсина, стоит упомянуть о таких диффузных болезнях соединительной ткани, при которых эмфизема встречается примерно у каждого 10-го больного (синдром Марфана, синдром Элерса-Данлоса) [51].

Продолжают изучать семейную предрасположенность к развитию ХОБЛ. E.K.Silverman et al. [52] наблюдали 44 семьи, где встречалось данное заболевание с ранним началом, тяжелым течением, без дефицита $\alpha_{1}$-антитрипсина. Женщин в изучаемой группе было довольно много (79,6 \%). У обследуемых 1-й степени родства, по сравнению с контрольной группой, показатели ОФВ 1 и отношения $\mathrm{OФB}_{1}$ к форсированной жизненной емкости легких (ФЖЕЛ) были снижены, несмотря на одинаковый индекс курения. У некурящих показатели не были снижены, гендерные различия не выявлены. Однофакторный анализ среди родственников 1-й степени, бросивших или продолжающих курить, показал, что у женщин, по сравнению с мужчинами, соотношение ОФВ 1 / ФЖЕЛ было значительно ниже $(81,4 \%$ vs 87,0 \%), а броходилатационный ответ существенно выше (прирост ОФВ 1 от исходного значения $-7,7 \%$ vs 4,1 \%). Более того, по результатам многофакторного анализа, женщины оказались более склонны к снижению показателя ОФВ $1<40$ \% долж. и развитию гиперреактивности бронхов, нежели мужчины [53]. Эти данные подтверждают влияние генетической предрасположенности женщин к развитию ХОБЛ.

Помимо генетической предрасположенности, среди факторов риска развития ХОБЛ стоит упомянуть питание и социоэкономический статус. Хотя до конца не ясно, является ли питание независимым фактором риска развития обструктивных нарушений, известно, что дефицит питательных веществ и недостаточная масса тела могут приводить к снижению силы и выносливости дыхательной мускулатуры, главным образом за счет потери массы респираторных мышц и снижения силы мышечных волокон [54]. Повсеместно женщины подвергаются большему риску недоедания и дефицита питательных веществ. По ориентировочным оценкам, порядка 20-45 \% женщин детородного возраста не получают рекомендованной ВО3 нормы суточной калорийности. Порочный круг замыкается, когда у плохо питающихся женщин рождаются дети с низкой массой тела. 
Можно не согласиться с тем, что социоэкономический статус относится только к эндогенным факторам, и продолжать рассматривать его в качестве фактора окружающей среды, однако есть основания полагать, что риск развития ХОБЛ находится в обратной зависимости от уровня жизни человека. Известно, что ХОБЛ превалирует у лиц с низким социоэкономическим статусом. Неясно, какие его признаки являются наиболее существенными, но в бедности или нужде пребывают $70 \%$ женщин, по сравнению с $30 \%$ мужчин [55].

Итак, к основным факторам риска развития и прогрессирования ХОБЛ можно отнести курение, загрязнение окружающей среды, эндогенные факторы, включая генетические особенности и аномалии, гиперреактивность бронхов и гормональный фон.

Прежде чем перейти к обсуждению гендерных особенностей в клинической картине и течении заболевания, не лишним будет обратить внимание на особенности анатомии и физиологии дыхательных путей у женщин.

Еще во время внутриутробного развития созревание легочной ткани, включая образование сурфактант-фосфолипидной системы, у девочек начинается на 10 дней раньше и происходит быстрее [56, 57], в связи с чем после рождения они менее подвержены развитию респираторного дистресс-синдрома и преходящего тахипноэ, чем мальчики. При рождении легкие девочек в среднем меньше и шире, чем у мальчиков и могут содержать меньшее количество респираторных бронхиол [58]. В течение жизни у женщин отмечается пропорциональный рост респираторного тракта и легочной паренхимы, в то время как у мужчин рост легочной паренхимы опережает рост дыхательных путей [47].

Несмотря на меньший размер легких, абсолютные и соответствующие диаметру значения форсированных экспираторных потоков, а также соотношение ОФВ 1 / ФЖЕЛ оказываются выше у женщин, чем у мужчин, на протяжении всей жизни [59-61]. Эти различия наиболее существенны в раннем детстве и несколько уменьшаются с возрастом, тогда как отношение остаточного объема легких к общей жизненной емкости легких увеличивается у девочек, но не у мальчиков. После 30 лет происходят процессы возрастного изменения легких, такие как уменьшение эластичности, снижение максимальных экспираторных потоков, нарастание остаточных объемов. Однако у женщин они начинаются несколько позже и прогрессируют медленнее, чем у мужчин.

Полагают, что основной причиной столь выраженных различий в анатомии и физиологии респираторного тракта является гормональное воздействие. Известно, что эстрогены и пролактин стимулируют развитие легких, а тестостерон замедляет его.

\section{Диагностика ХОБЛ и гендерные различия}

При наличии хронического кашля с продукцией мокроты или без нее, с упорно продолжающейся или прогрессирующей одышкой при указаниях в анам- незе на воздействие факторов риска заболевания в круг дифференциально-диагностического поиска обязательно должна включаться ХОБЛ [2]. Однако диагностика ХОБЛ остается недостаточной и ошибочной - зачастую определяется другое заболевание, особенно у женщин.

K.R.Chapman et al. [62] провели исследование в США и Канаде, в котором проанализировали работу врачей первичного звена, и обнаружили, что ХОБЛ чаще диагностируется у мужчин, чем у женщин. Врачам представляли сценарии гипотетических случаев. Одной их половине говорили, что гипотетический пациент женского пола, другой - что пациент мужского пола. Врачей просили поставить наиболее вероятный диагноз, если известно, что пациент - курильщик, страдает от одышки и кашля. После опроса и физикального обследования больных ХОБЛ была заподозрена у 64,6 \% мужчин и $49 \%$ женщин. После предоставления объективной информации данных спирометрии - это заболевание было диагностировано уже у $74 \%$ мужчин и 64,6\% женщин (разница статистически незначима). На следующем этапе исследования врачам сообщали о том, что гипотетический пациент прошел 2-недельный курс лечения пероральными глюкокортикостероидами (ГКС) без эффекта, после чего гендерная погрешность нивелировалась: ХОБЛ была распознана у 85,4 \% мужчин и 78,1 \% женщин. Исследование показало, что проведение спирометрии снижает риск гиподиагностики заболевания, особенно у женщин.

Спирометрия является золотым стандартом диагностики ХОБЛ и мониторирования прогрессирования заболевания, однако на практике ее проводят недостаточно часто. В вышеописанном исследовании K.R.Chapman et al. [62] только $22 \%$ врачей первичного звена назначили исследование легочной функции.

N.R.Anthonisen et al. [63] оценили частоту назначения спирометрии пациентам с обструктивными болезнями легких старше 35 лет: она варьировалась от $27 \%$ у больных, посещавших врача 1 раз в год, до $53 \%$ среди пациентов, у которых число визитов к врачу в год превышало 19. При расчете гендерной погрешности оказывается, что женщинам с меньшей вероятностью назначают спирометрию: отношение шансов составляет 0,84 для женщин по сравнению с мужчинами (95\%-ный доверительный интервал - 0,72-0,98) [64]. Без спирометрии женщинам с симптомами ХОБЛ вместо этого может быть поставлен диагноз БА.

\section{Клиническая картина ХОБЛ и гендерные различия}

Особенность клинической картины ХОБЛ - в том, что долгое время болезнь протекает без выраженных клинических проявлений, и это затрудняет своевременную постановку диагноза.

Для большинства пациентов диагностика ХОБЛ начинается с сообщения лечащему врачу о проявлениях, связанных с системой органов дыхания, одышке, кашле, выделении мокроты, хрипах, ошуще- 
нии стеснения в грудной клетке, а также о сопутствующих состояниях - беспокойстве, депрессии, потере в весе.

В серии исследований изучались гендерные различия симптомов (таблица) [65]. Отмечено, что женщины, страдающие ХОБЛ, с той же степенью бронхиальной обструкции, что и у мужчин, чаще сообщают об одышке [64, 66], но реже - о выделении мокроты $[64,68]$. Кроме того, женщины с ХОБЛ чаще испытывают депрессию, беспокойство, усталость по сравнению с мужчинами, даже после сопоставления по показателям функции легких [67, 69]. Данные относительно гендерных различий связанного со здоровьем качества жизни (СЗКЖ) оказались менее последовательны. В одних исследованиях СЗКЖ было хуже у женщин $[66,71,72]$, в других - в течение длительного времени сильнее снижалось у мужчин [72], либо гендерные различия СЗКЖ вообще отсутствовали [74].

Описано 2 основных фенотипа заболевания: эмфизематозный и бронхитический. Опираясь на данные Национального испытания по лечению эмфиземы NETT, собравшего > 1500 больных, направленных на хирургическую операцию резекции объема легких, F.J.Martinez et al. пришли к выводу, что среди женщин преобладает 2-й фенотипический вариант ХОБЛ [46]. В ходе анализа компьютерных томограмм грудной клетки и гистологического исследования ткани легкого обнаружено, что у женщин, страдающих ХОБЛ тяжелого течения, анатомически меньшие просветы дыхательных путей сочетаются с непропорционально более выраженным утолщением стенок мелких бронхов, по сравнению с мужчинами. Кроме того, эмфизема у них менее обширная, характеризуется меньшим размером отверстий и меньшим периферическим участием.

Тяжесть заболевания можно оценивать не только по клиническим симптомам, но и по частоте госпитализаций. Показано, что риск госпитализации по поводу ХОБЛ у женщин выше, чем у мужчин: в 1,5 раза (относительный риск (ОР) - 1,5-2,1), по данным исследования CCHS, и в 3,6 раза (OP 1,4-9,0), по данным GPS [27]. Более тяжелое течение заболевания у женщин согласуется с данными о большей их предрасположенности к развитию ХОБЛ и возникновением его в более раннем возрасте, по сравнению с мужчинами. Однако высокая внутрибольничная смертность среди женщин, страдающих ХОБЛ, не выявлена.

Развитие осложнений также усугубляет течение ХОБЛ. Одним из наиболее распространенных из них, встречающимся преимущественно у женщин, является остеопороз. Хорошо известно, что его частота довольно высока у больных ХОБЛ, принимающих системные ГКС и большие дозы ингаляционных ГКС [75, 76]. Однако даже у больных ХОБЛ, не использовавших эти препараты, частота развития остепороза составила 50 \%, что вдвое выше, чем среди лиц той же возрастной категории, но не страдающих ХОБЛ [77].

\section{Лечение ХОБЛ и гендерные различия}

Одной из главных целей терапии ХОБЛ является предотвращение дальнейшего ухудшения легочной

Таблица

Гендерные различия симптомов ХОБЛ

\begin{tabular}{|c|c|c|c|}
\hline Симптомы & Исследование & Дизайн и проект исследования & Гендерные различия симптоматики \\
\hline \multirow[t]{3}{*}{ Одышка } & $\begin{array}{l}\text { J.P.de Torres } \\
\text { et al. [66] }\end{array}$ & $\begin{array}{l}\text { Перекрестное; } 53 \text { мужчины и женщины с ХОБЛ, набранные } \\
\text { по показателям функции легких из клиники болезней легких }\end{array}$ & $\begin{array}{l}\text { Женщины с ХОБЛ сообщают об одышке } \\
\text { (по шкале ММRС) чаще, чем мужчины; } p=0,003\end{array}$ \\
\hline & $\begin{array}{l}\text { L.Watson } \\
\text { et al. }[64]\end{array}$ & $\begin{array}{l}\text { Перекрестное; телефонный опрос населения методом } \\
\text { случайной выборки; самоотчет о диагнозе ХОБЛ }\end{array}$ & $\begin{array}{l}\text { Женщины сообщают о тяжелой одышке } \\
\text { (по шкале MRC) чаще, чем мужчины; } p<0,05\end{array}$ \\
\hline & $\begin{array}{l}\text { F.Di Marco } \\
\text { et al. [67] }\end{array}$ & $\begin{array}{l}\text { Метод случай-контроль; распространенность симптомов } \\
\text { у больных ХОБЛ, посещающих клинику болезней легких, } \\
\text { в сравнении с пациентами без ХОБЛ }\end{array}$ & $\begin{array}{l}\text { Женщины с ХОБЛ сообщают об одышке } \\
\text { (по шкале MRC) чаще, чем мужчины; } p=0,003\end{array}$ \\
\hline $\begin{array}{l}\text { Хронический } \\
\text { кашель }\end{array}$ & $\begin{array}{l}\text { L.Watson } \\
\text { et al. [68] }\end{array}$ & $\begin{array}{l}\text { Длительное рандомизированное клиническое } \\
\text { исследование применения будесонида, ветвь плацебо }\end{array}$ & $\begin{array}{l}\text { Более высокая распространенность "разбуженных } \\
\text { приступом кашля" (p<0,001) и "хронического } \\
\text { кашля зимой" (p=0,03) среди женщин, } \\
\text { по сравнению с мужчинами }\end{array}$ \\
\hline \multirow[t]{2}{*}{$\begin{array}{l}\text { Выделение } \\
\text { мокроты }\end{array}$} & $\begin{array}{l}\text { R.K.Cydulka } \\
\text { et al. [69] }\end{array}$ & $\begin{array}{l}\text { Вторичный анализ проспективной когорты пациентов, } \\
\text { поступающих в отделение скорой помощи с внезапным } \\
\text { обострением ХОБЛ }\end{array}$ & $\begin{array}{l}\text { Мужчины чаще, чем женщины, сообщают о кашле } \\
\text { на протяжении большого количества дней; } p=0,02\end{array}$ \\
\hline & $\begin{array}{l}\text { L.Watson } \\
\text { et al. [64] }\end{array}$ & $\begin{array}{l}\text { Перекрестное; телефонный опрос населения методом } \\
\text { случайной выборки; самоотчет о диагнозе ХОБЛ }\end{array}$ & $\begin{array}{l}\text { Мужчины и женщины одинаково часто сообщают } \\
\text { о мокроте }\end{array}$ \\
\hline Депрессия & $\begin{array}{l}\text { F.Di Marco } \\
\text { et al. [67] }\end{array}$ & $\begin{array}{l}\text { Метод случай-контроль; распространенность симптомов } \\
\text { у пациентов с ХОБЛ, посещающих клинику болезней } \\
\text { легких, в сравнении с пациентами без ХОБЛ }\end{array}$ & $\begin{array}{l}\text { Высокая распространенность депрессии по } \\
\text { сравнению с контрольной группой; у женщин выше } \\
\text { уровень депрессии и хуже качество жизни, } \\
\text { связанное с симптомами, чем у мужчин }\end{array}$ \\
\hline \multirow[t]{2}{*}{$\begin{array}{l}\text { Беспокойство } \\
\text { и усталость }\end{array}$} & $\begin{array}{l}\text { F.Di Marco } \\
\text { et al. [67] }\end{array}$ & $\begin{array}{l}\text { Метод случай-контроль; распространенность симптомов } \\
\text { у пациентов с ХОБЛ, посещающих клинику болезней } \\
\text { легких, в сравнении с пациентами без ХОБЛ }\end{array}$ & $\begin{array}{l}\text { Высокая распространенность беспокойства по } \\
\text { сравнению с контрольной группой; у женщин уровень } \\
\text { беспокойства выше, по сравнению с мужчинами }\end{array}$ \\
\hline & $\begin{array}{l}\text { A.G.Gift, } \\
\text { C.E.Shepard } \\
{[70]}\end{array}$ & $\begin{array}{l}\text { Перекрестное; исследование пациентов, посещающих } \\
\text { клинику болезней легких }\end{array}$ & $\begin{array}{l}\text { Психологические симптомы сходны у пациентов обоего } \\
\text { пола, за исключением беспокойства, уровень которого } \\
\text { выше у женщин. Женщины сообщают о более } \\
\text { выраженной усталости }\end{array}$ \\
\hline
\end{tabular}


функции. Здесь существуют несколько направлений, основными из них являются снижение влияния факторов риска, проведение образовательных программ, медикаментозное и немедикаментозное лечение.

Борьба с табакокурением как основным фактором риска развития ХОБЛ относится к важнейшим задачам. Прекращение курения является единственным наиболее эффективным и экономически обоснованным способом, позволяющим не только сократить риск развития заболевания, но и предотвратить его прогрессирование [2, 78]. К настоящему времени доказанной эффективностью обладают только 2 метода - никотинозаместительная терапия и консультирование больного врачами и средним медперсоналом (беседы).

Анализ 2 продольных исследований показал, что отказ от курения приводит к 50\%-ному снижению хронического кашля, выделения мокроты и приступов одышки. Как у мужчин, так и у женщин употребление большего количества сигарет в сутки и начало курения в ранние годы увеличивает риск сохранения этих симптомов на протяжении еще нескольких лет после прекращения курения [79].

Проспективное рандомизированное многоцентровое исследование здоровья легких LHS показало, что отказ от табакокурения при наличии ранних симптомов бронхиальной обструкции и сниженных экспираторных потоках ведет к прекращению падения ОФВ 180$]$. В конце 1-го года в группе пациентов, получавших никотинозаместительную терапию с использованием никотиносодержащей жевательной резинки, ОФВ 1 увеличивался (в среднем на 47 мл, или $2 \%$ долж.), в то время как в контрольной группе он продолжал падать. Причем у женщин, страдающих ХОБЛ и бросивших курить, за 1-й год достигнуто улучшение $\mathrm{OФB}_{1}$, в 2,3 раза превышающее таковое у мужчин (3,7 \% долж. vs 1,6 \% долж. соответственно). Однако при продолжении табакокурения ежегодное падение ОФВ 1 оказалось достоверно больше у женщин по сравнению с мужчинами [81].

Несмотря на известное больным значение отказа от курения, доля пациентов обоего пола, прекративших курить, остается небольшой. Более того, среди женщин этот процент еще ниже [82-84]. Причин этому много, но до конца они так и не выяснены. К физиологическим факторам, предрасполагающим женщин реже отказываться от курения, относят измененную чувствительность и толерантность к никотину, что ведет к меньшей эффективности никотинозамещающей терапии, а также более выраженные, чем у мужчин-курильщиков, симптомы отмены и негативное влияние менструального цикла на попытки отказа от курения [85]. Среди поведенческих и психологических причин выделяют боязнь прибавки в весе, слабую поддержку окружающих и больший, чем у мужчин, соблазн покурить с друзьями, повышенную дисфорию и развитие депрессии при отказе от курения. Все эти факторы в совокупности приводят к тому, что женщинам труднее, чем мужчинам, длительно воздерживаться от табака.
Прибавка в весе - одно из самых распространенных последствий при отказе от курения. В исследовании LHS показано, что в 1-й год после прекращения курения женщины прибавляют в весе в среднем 5,2 кг, в то время как мужчины - 4,9 кг. А к концу периода наблюдения 19,1\% женщин и 7,6 \% мужчин весят как минимум на $20 \%$ больше, чем исходно [86]. Впоследствии влияние на показатели легочной функции каждого набранного килограмма после отказа от курения более выражено у мужчин, чем у женщин (за 5 лет падение ОФВ 1 - на 11,1 мл/кг vs 5,6 мл/кг, падение ЖЕЛ - на 17,4 мл/кг vs 10,6 мл/кг у мужчин и женщин соответственно) [87]. Однако по сравнению с положительным влиянием на легочную функцию прибавка в весе после прекращения курения не столь значима.

По всем аспектам лечения заболевания проводится обучение, которое может осуществляться в разных формах: консультации врача или других медицинских работников, домашние программы, занятия вне дома, полноценные программы легочной реабилитации.

В соответствии с международными и отечественными клиническими рекомендациями по ХОБЛ [2, 78], медикаментозное лечение больных с ХОБЛ определяется стадией заболевания, тяжестью симптомов, выраженностью бронхиальной обструкции, наличием дыхательной или правожелудочковой недостаточности, сопутствующими заболеваниями. В нескольких исследованиях изучались особенности терапии в зависимости от его пола. Было отмечено, что на лечении находилось вдвое больше женщин с ХОБЛ легкого и среднетяжелого течения, по сравнению с мужчинами. Однако в схемах медикаментозной терапии у больных с ХОБЛ тяжелого течения гендерные различия не выявлены [88].

К основным лекарственным средствам, применяемым при ХОБЛ, относят бронходилататоры $\left(\beta_{2}\right.$-адренергические, антихолинергические препараты и теофиллины) и ГКС. Большинство из них рекомендуется принимать посредством дозированных аэрозольных ингаляторов (ДАИ), имеющих неоспоримые преимущества. Однако у больных ХОБЛ часто встречается неправильная техника ингаляций проблема, приводящая к плохой доставке лекарства в дыхательные пути, снижающая контроль над болезнью и увеличивающая частоту применения ингаляторов. Так, меньше 80 \% пациентов могут эффективно использовать ДАИ даже после инструкции медицинских работников, хотя эти ингаляторы назначаются чаще всего [89]. По мнению D.E.Goodman et al. [90], у женщин ошибки в технике ингаляции встречаются чаще, чем у мужчин, однако причины этого неясны. В данном исследовании лишь $43 \%$ мужчин и $4 \%$ женщин смогли корректно применить ДАИ при электронном контролировании потоков, скорости и временных интервалов, свойственных правильному ингаляционному маневру.

Накоплено относительно немного информации о гендерных различиях ответа на терапию. В 2 разных исследованиях воздействие комбинированной терапии сальметеролом / флутиказона пропионатом 
или тиотропиума бромидом на функцию легких, симптомы и качество жизни было схожим у мужчин и женщин [91, 92]. В рандомизированном плацебоконтролируемом исследовании, в котором изучалось влияние флутиказона пропионата на реактивность бронхов, после 6-недельного курса лечения оказалось, что наиболее значимый эффект ГКС был достигнут у мужчин, а не у женщин, и он не зависел от стажа курения, возраста или ОФВ 1 [93].

В последние 5 лет активно проводятся клинические исследования лекарственных средств со сверхдлительным действием, эффективность которых сохраняется в течение 24 ч после 1-кратного применения (тиотропий, индакатерол и другие). В октябре 2008 г. на XVIII Конгрессе Европейского респираторного общества были представлены результаты рандомизированного двойного слепого плацебоконтролируемого исследования UPLIFT (Understarding Potential Long-term Impacts on Function with Tiotropium) [94]. UPLIFT является одним из самых крупных из когда-либо предпринятых исследований ХОБЛ. Оно проводилось в 37 странах мира в течение 4 лет, и в нем приняли участие 5993 пациента. Главной его целью стало определение влияния тиотропия бромида на замедление падения ОФВ 1 . Больных рандомизировали на 2 группы: 1-й группе в дополнение к основной терапии назначили тиотропий в дозе 18 мкг 1 раз в сутки, пациенты 2-й группы получали плацебо. Всем больным не разрешался прием короткодействующих ингаляционных антихолинергических препаратов. Средний возраст пациентов составил $65 \pm 8$ лет, 30 \% были активными курильщиками, среди участников было 1498 женщин (25\%).

В ходе исследования тиотропий продемонстрировал очень хорошие отдаленные результаты: он уменьшает падение $\mathrm{OФB}_{1}$, снижает риск обострений, ведущих к госпитализации (риск по отношению к контрольной группе $-0,86 ; p<0,001)$ и количество обострений в расчете на пациента в год (14\%; $p<0,001)$, значительно увеличивает время до наступления 1-го обострения ХОБЛ (в среднем на 4,1 мес.; $p<0,001)$, улучшает качество жизни пациентов, согласно респираторному опроснику Госпиталя св. Георгия $(p<0,001)$. Кроме того, в группе тиотропия отмечалось статистически значимое снижение смертности от всех причин на $16 \%(p=0,016)$, улучшение функции легких после 4 лет лечения $(p<0,001)$, сокращение летальности от респираторных $(p<0,05)$ и сердечно-сосудистых $(p<0,05)$ причин [95]. К сожалению, выборочная оценка влияния тиотропия отдельно на мужчин и женщин не проводилась. По словам M.Decramer, ведущего исследователя UPLIFT и профессора медицины госпиталя Католического университета Левена (Бельгия), благодаря результатам исследования возрастает понимание того, "как лечение может повлиять на клиническое течение ХОБЛ. Это позволит врачам раньше и с большей уверенностью начинать длительную поддерживающую терапию ХОБЛ".

Изучается эффективность и безопасность индакатерола ( $\beta_{2}$-агонист с длительностью действия 24 ч) при хронических обструктивных заболеваниях. Уже проведено 26 исследований (из них 19 рандомизированных плацебо-контролируемых), и еще 8 - продолжаются. В рамках завершившихся исследований индакатерол в дозе от 23 до 3000 мкг в сутки получали 1002 больных БА и 853 пациента с ХОБЛ. По обобщенным данным всех работ, доля женщин составила 24-41 \% за первые 3 мес., 24-37 \% - за 6 мес. и 18-23\% - за 12 мес. исследований. Однако о результатах влияния данного препарата на пациентов женского пола не сообщается.

K немедикаментозным методам терапии относят хирургическое лечение и реабилитацию больных. Хирургическое лечение ХОБЛ, получившее широкое распространение за последние 10-летия в различных странах мира, является одним из новых и важных направлений в хирургии болезней органов дыхания. Поскольку число больных ХОБЛ превышает реальные возможности трансплантологии, в 1995 г. J.D.Cooper et al. [96] начали выполнять 2-сторонние краевые резекции легких у пациентов с тяжелой эмфиземой, ожидающих операции по трансплантации легких. Этот вид вмешательств назвали хирургической редукцией объема легкого (ХРОЛ). Поскольку диффузная эмфизема - прогрессирующее заболевание, послеоперационное улучшение носит временный характер. Достигая своего пика через 12 мес. после операции, показатели внешнего дыхания, бодиплетизмографии и одышки постепенно начинают возвращаться к исходным значениям. Поэтому оперативную коррекцию легочного объема относят к паллиативным хирургическим вмешательствам.

Исследовательская группа Национального испытания по лечению эмфиземы NETT [97] проанализировала когорту рандомизированных из 14 американских центров пациентов, которым была предписана ХРОЛ. Исследуемая группа включала в себя 1053 больных, среди которых было 38,8 \% женщин. Целью исследования стало выявление гендерных различий при тяжелой эмфиземе легких. Оказалось, что, по сравнению с мужчинами, женщины, направленные на ХРОЛ, были немного моложе, сообщали о значительно более короткой продолжительности курения и более старшем возрасте ко времени его начала. У них были ниже индекс массы тела и толерантность к физической нагрузке, а обструкция дыхательных путей - более выражена. Для определенного должного значения ОФВ 1 , возраста, индекса курения, выраженности эмфиземы женщины имели более высокий модифицированный показатель BODE (индекс массы тела, обструкция, диспноэ, физическая нагрузка), в большей степени испытывали одышку и депрессию. При анализе результатов опросника SF-36 у женщин выявлены более низкие показатели самочувствия и умственной способности, по сравнению с мужчинами [46].

При ХОБЛ всех стадий высокоэффективной оказывается терапевтическая программа, включающая в себя курсы физических тренировок, коррекцию массы тела, обучение и психосоциальную поддержку. Легочная реабилитация приводит к уменьшению 
степени одышки, улучшению легочной функции, СЗКЖ, сокращению частоты госпитализаций, снижению беспокойства и депрессии. Так как большинство исследований в этой области проводится среди пациентов мужского пола, о воздействии легочной реабилитации на женщин информации немного.

M.C.Haggerty et al. [98] оценивали улучшение различных показателей на фоне легочной реабилитации у 164 больных ХОБЛ, среди которых было 54 \% женщин. Оказалось, что у них регистрировались более выраженные улучшения в блоке "Влияние и эмоции" в Опроснике по хроническим респираторным заболеваниям (Chronic Respiratory Disease Questionnaire, CRDQ) и психологическом блоке Шкалы состояния функции легких (Pulmonary Function Status Scale, PFSS), по сравнению с мужчинами. Но остальные показатели CRDQ и PFSS, а также пройденное расстояние в 6-минутном шаговом тесте были одинаковыми у участников исследования обоих полов. Учитывая, что реабилитационные программы были всесторонними, неясно, какой аспект внес больший вклад.

C.G.Foy et al. обнаружили, что после короткой 3-месячной программы физических упражнений качество жизни стало выше как у женщин, так и у мужчин [99]. При продлении тренировок до 18 мес. у мужчин продолжало улучшаться СЗКЖ, тогда как у женщин дополнительного положительного воздействия не было.

\section{Заключение}

На сегодняшний день ХОБЛ, наряду с артериальной гипертонией, ишемической болезнью сердца и сахарным диабетом, входит в группу хронических заболеваний, на долю которых приходится около $1 / 3$ среди всех других форм патологии человека. По прогнозам мировых исследователей, уровень заболеваемости ХОБЛ будет продолжать расти, и особенно заметно - среди женщин. В связи с этим в будущем следует обращать больше внимания на особенности диагностики, клинического течения и лечения женщин, страдающих ХОБЛ, и углубить научные исследования в этом направлении.

\section{Литература}

1. Mannino D.M., Buist A.S. Global burden of COPD: risk factors, prevalence, and future Trends. Lancet 2007; 370: 765-773.

2. Global Initiative for Chronic Obstructive Lung Disease. Global Strategy for the Diagnosis, Management and Prevention of Chronic Obstructive Pulmonary Disease. Updated 2007 (www. goldcopd.com).

3. Celli B.R., MacNee W., Agusti A.G. et al. Standards for the diagnosis and treatment of patients with COPD: a summary of the ATS / ERS position paper. Eur. Respir. J. 2004; 23: 932-946.

4. A report of the task force on the NIH's women's health research agenda for the twenty-first century. Bethesda, MD: National Institutes of Health; 1999. NIH Publication No. 99-4385.
5. Чучалин А.Г. Белая книга. Пульмонология. Россия 2003. M.; 2003.

6. http://nhlbi.nih.gov/health/public/lung/other/copd_fact.htm

7. Soriano J.R., Maier W.C., Egger P. et al. Recent trends in physician diagnosed COPD in women and men in the UK. Thorax 2000; 55: 789-794.

8. Feenstra T.L., van Genugten M.L., Hoogenveen R.T. et al. The impact of ageing and smoking on the future burden of chronic obstructive pulmonary disease: a model analysis in the Netherlands. Am. J. Respir. Crit. Care Med. 2001; 164: 590-596.

9. Miller J.D., Foster T., Boulanger L. et al. Direct costs of COPD in the U.S.: an analysis of Medical Expenditure Panel Survey (MEPS) data. COPD 2005; 2: 311-318.

10. Jemal A., Ward E., Hao Y., Thun M. Trends in the leading causes of death in the United States, 1970-2002. J. A. M. A. 2005; 294: 1255-1259.

11. Brett K.M., Hayes S.G. Women's health and mortality chartbook. Washington, DC: DHHS Office on Women's Health; 2004.

12. Mathers D.M., Loncar D. Projections of global mortality and burden of disease from 2002 to 2030. PLoS Med. 2006; 3: e442.

13. Chapman K.R. Chronic obstructive pulmonary disease: are women more susceptible than men? Clin. Chest Med. 2004; 25: $331-341$.

14. Mannino D.M., Homa D.M., Akinbami L.J. et al. Chronic obstructive pulmonary disease surveillance: United States, 1971-2000. MMWR Surveill. Summ. 2002; 51: 1-16.

15. Vestbo J. Epidemiology. In: Voelkel N.F., MacNee W., eds. Chronic obstructive lung disease. Hamilton, Canada: BC Decker Inc.; 2002. 41-55.

16. Esson K., Leeder S.R. The millennium development goals and tobacco control: an opportunity for global partnership. Geneva: World Health Organization; 2004.

17. World Health Organization. Gender, health, and tobacco. Geneva: World Health Organization; 2003.

18. Ernster $V$. Women and the tobacco epidemic: challenges for the 21st century. Geneva: World Health Organization; 2001.

19. Mackay J., Amos A. Women and tobacco. Respirology 2003; 8: $123-130$.

20. The European tobacco control report 2007. Jeneva: World Health Organization, 2007; 153.

21. Герасименко Н.Ф., Заридзе Д.Г., Сахарова Г.М. Здоровье или табак. М.: Форум "Здоровье или табак"; 2007.

22. Mollerup S., Ryberg D., Hewer A. et al. Sex differences in lung CYP1A1 expression and DNA adduct levels among lung cancer patients. Cancer Res. 1999; 59: 3317-3320.

23. Han W., Pentecost B.T., Pietropaolo R.L. et al. Estrogen receptor alpha increases basal and cigarette smoke extractinduced expression of CYP1A1 and CYP1B1, but not GSTP1, in normal human bronchial epithelial cells. Mol. Carcinog. 2005; 44: 202-211.

24. Benowitz N.L., Lessov-Schlaggar C.N., Swan G.E., Jacob P. III. Female sex and oral contraceptive use accelerate nicotine metabolism. Clin. Pharmacol. Ther. 2006; 79: 480-488.

25. Xu X., Li B., Wang $L$. Gender difference in smoking effects on adult pulmonary function. Eur. Respir. J. 1994; 7: 477-483.

26. Хи X., Weiss S.T., Rijcken B., Shouten J.P. Smoking, changes in smoking habits, and rates of decline in $\mathrm{FEV}_{1}$ : new insight into gender differences. Eur. Respir. J. 1994; 7: 1056-1061.

27. Prescott E., Bjerg A.M., Andersen P.K. et al. Gender difference in smoking effects on lung function and risk of hospi- 
talization for COPD: results from a Danish longitudinal study. Eur. Respir. J. 1997; 10 (4): 822-827.

28. Carter R., Nicotra B., Huber G. Differing effects of airway obstruction on physical work capacity and ventilation in men and women with COPD. Chest 1994; 106 (6): 1730-1739.

29. Camilli A.E., Burrows B., Knudson R.J. et al. Longitudinal changes in forced expiratory volume in one second in adults. Am. Rev. Respir. Dis. 1987; 135: 794-799.

30. Peat J.K., Woolcock A.J., Cullen K. Decline of lung function and development of chronic airflow limitation: a longitudinal study of smokers and non-smokers in Busselton. Western Australia. Thorax 1990; 45: 32-37.

31. Anthonisen N.R., Connet J.E., Murray R.P. for the Lung Health Study Research Group. Smoking and lung function of lung health study participants after 11 years. Am. J. Respir. Crit. Care Med. 2002; 166: 675-679.

32. de Torres J., Campo A., Casanova C. et al. Gender and chronic obstructive pulmonary disease in high-risk smokers. Respiration 2006; 73: 306-310.

33. Birring S.S., Brightling C.E., Bradding P. et al. Clinical, radiological, and induced sputum features of chronic obstructive pulmonary disease in nonsmokers. Am. J. Respir. Crit. Care Med. 2002; 166: 1078-1083.

34. He J., Gu D., Wu X. et al. Major causes of death among men and women in China. N. Engl. J. Med. 2005; 353: 1124-1134.

35. Ramirez-Venegas A., Sansores R.H., Perez-Padilla R. et al. Survival of patients with chronic obstructive pulmonary disease due to biomass smoke and tobacco. Am. J. Respir. Crit. Care Med. 2006; 173: 393-397.

36. Dockery D.W., Pope C.A., Xu X. et al. An association between air pollution and mortality in six US cities. N. EngI. J. Mod. 1993; 329: 1753-1759.

37. Tao X., Hong C.J., Yu S. et al. Priority among air pollution factors for preventing chronic obstructive pulmonary disease in Shanghai. Sci. Total Environ. 1992; 127: 57-67.

38. Pandey M.R. Domestic smoke pollution and chronic bronchitis in a rural community of the Hill Region of Nepal. Thorax 1984; 39: 337-339.

39. Amoli K. Bronchopulmonary disease in Iranian housewives chronically exposed to indoor smoke. Eur. Respir. J. 1998; 11: 659-663.

40. Dossing M., Khan J., al-Rabiah F. Risk factors for chronic obstructive lung disease in Saudi Arabia. Respir. Med. 1994; 88: 519-522.

41. Perez-Padilla R., Regalado U., Vedal S. et al. Exposure to biomass smoke and chronic airway disease in Mexican women. Am. J. Respir. Crit. Care Med. 1996; 154: 701-706.

42. Gan W.Q., Man S.F., Postma D.S. et al. Female smokers beyond the perimenopausal period are at increased risk of chronic obstructive pulmonary disease: a systematic review and meta-analysis. Respir. Res. 2006; 7: 52.

43. Kanner R.E., Connett J.E., Altose M.D. et al. Gender difference in airway hyperresponsiveness in smokers with mild COPD: the Lung Health Study. Am. J. Respir. Crit. Care Med. 1994; 150: 956-961.

44. Paoletti P., Carrozzi L., Viegi G. et al. Distribution of bronchial responsiveness in a general population: effect of sex, age, smoking, and level of pulmonary function. Am. J. Respir. Crit. Care Med. 1995; 151: 1770-1777.

45. Rijken B., Schouten J.P., Xu X. et al. Airway hyperresponsiveness to hystamine is associated with accelerated decline of $\mathrm{FEV}_{1}$. Am. J. Respir. Crit. Care Med. 1995; 151: 1377-1382.
46. Martinez F.J., Curtis J.L., Sciurba F. et al. Sex differences in severe pulmonary emphysema. Am. J. Respir. Crit. Care Med. 2007; 176: 243-252.

47. Becklake M.R., Kauffmann F. Gender differences in airway behaviour over a human life span. Thorax 1999; 54: 1119-1138.

48. Kelly R.W., Illingworth P., Baldie G. et al. Progesteron control of interleukin-8 production in endometrium and chorio-decidual cells underlines the role of the neutrophil in menstruation and parturition. Hum. Reprod. 1994; 9: 253-258.

49. Saetta M., Turato G., Maestrelli P. et al. Cellular and structural bases of chronic obstructive pulmonary disease. Am. J. Respir. Crit. Care Med. 2001; 163: 1304-1309.

50. Kauffmann T., Becklake M.R. Sex and gender. Eur. Respir. Mon. 2000; 15: 288-304.

51. Lee P., Gildea T.R., Stoller J.K. Emphysema in nonsmokers: alpha-1-antitrypsin deficiency and other causes. Cleveland Clin. J. Med. 2002; 69: 928-946.

52. Silverman E.K., Weiss S.T., Drazen J.M. et al. Genetic epidemiology of severe, early-onset chronic obstructive pulmonary disease: risk to relatives for airflow obstruction and chronic bronchitis. Am. J. Respir. Crit. Care Med. 1998; 157: 1770-1778.

53. Silverman E.K., Weiss S.T., Drazen J.M. et al. Gender-related differences in severe, early-onset chronic obstructive pulmonary disease. Am. J. Respir. Crit. Care Med. 2000; 162: 2152-2158.

54. Wilson D.O., Rogers R.M., Wright E.C. et al. Body weight in chronic obstructive pulmonary disease. The National Institutes of Health Intermittent Positive-Pressure Breathing Trial. Am. Rev. Respir. Dis. 1989; 139: 1435-1438.

55. Varkey A. Cronic obstructive pulmonary disease in women: exploring gender differences. Curr. Opin. Pulm. Med. 2004; 10: $98-103$.

56. Torday J.S., Nielsen H.C. The sex difference in fetal lung surfactant production. Exp. Lung Res. 1987; 1: 1-19.

57. Fleisher B., Kulovich M.V., Hallman M., Gluck L. Lung profile: sex differences in normal pregnancy. Obstetr. and Gynecol. 1985; 66: 327-330.

58. Thurlbeck W.M. Postnatal human lung growth. Thorax 1982; 37: 564-571.

59. Quanjer P.H., Borsboom G.J., Brunekreff B. et al. Spirometric reference values for white European children and adolescents: Polgar revisited. Pediatr. Pulmonol. 1995; 19: $135-142$.

60. Clausen J.L., Coates A.L., Quanjer P.H. Measurement of lung volumes in humans: review and recommendations from an ATS / ERS workshop. Eur. Respir. J. 1997; 10: $1205-1206$.

61. American Thoracic Society. Lung function testing: selection of reference values and interpretative strategies. Am. Rev. Respir. Dis. 1991; 144: 1202-1218.

62. Chapman K.R., Tachkin D.P., Pye D.J. Gender bias in the diagnosis of COPD. Chest 2001; 119: 1691-1695.

63. Anthonisen N.R., Wooldrage K., Manfreda J. Use of spirometry and respiratory drugs in Manitobans over 35 years of age with obstructive lung diseases. Can. Respir. J. 2005; 12: 69-74.

64. Watson L., Vestbo J., Postma D.S. et al. Gender differenses in the management and experience of chronic obstructive pulmonary disease. Respir. Med. 2004; 98: 1207-1203.

65. Camp P.G., Goring S.M. Gender and the diagnosis, management, and surveillance of chronic obstructive pulmonary disease. Proc. Am. Thorac. Soc. 2007; 4: 686-691. 
66. de Torres J.P., Casanova C., Hernandez C. et al. Gender and COPD in patients attending a pulmonary clinic. Chest 2005; 128: 2012-2016.

67. Di Marco F., Verga M., Reggente M. et al. Anxiety and depression in COPD patients: the roles of gender and disease severity. Respir. Med. 2006; 100: 1767-1774.

68. Watson L., Vonk J.M., Lofdahl C.G. et al. Predictors of lung function and its decline in mild to moderate COPD in association with gender: results from the EUROSCOP study. Respir. Med. 2006; 100: 746-753.

69. Cydulka R.K., Rowe B.H., Clark S. et al. Gender differences in emergency department patients with chronic obstructive pulmonary disease exacerbations. Acad. Emerg. Med. 2005; 12: 1173-1179.

70. Gift A.G., Shepard C.E. Fatigue and other symptoms in patients with chronic obstructive pulmonary disease: do women and men differ? J. Obstetr. Gynecol. Neonat. Nurs. 1999: 28: 201-208.

71. Katzura H., Yamada K., Wakagayashi R., Kida K. Genderassociated differences in dyspnea and health-related quality of life in patients with chronic obstructive pulmonary disease. Respirology 2007; 12: 427-432.

72. Low G., Gutman G. Examining the role of gender in healthrelated quality of life: perceptions of older adults with chronic obstructive pulmonary disease. J. Gerontol. Nurs. 2006; 21: 42-49.

73. Hesselink A.E., van der Windt P.A., Penninx B.W. et al. What predicts change in pulmonary function and quality of life in asthma or COPD? J. Asthma 2006; 43: 513-519.

74. Stahl E., Lindberg A., Jansson S.A. et al. Health-related quality of life is related to COPD severity. Hlth Qual. Life Outcomes 2005; 3: 56.

75. Renkema T.E., Schouten J.P., Koeter G.H., Postma D.S. Effects of long-term treatment with corticosteroids in COPD. Chest 1996; 109: 1156-1162.

76. Lee T.A., Weiss K.B. Fracture risk associated with inhaled corticosteroid use in chronic obstructive pulmonary disease. Am. J. Respir. Crit. Care Med. 2004; 169: 855-859.

77. Katsura H., Kida K. A comparison of bone mineral density in elderly female patients with COPD and bronchial asthma. Chest 2002; 122: 1949-1955.

78. Чучалин А.Г. (ред.). Хроническая обструктивная болезнь легких. (Федеральная программа): Практическое руководство для врачей. 2-е изд. М.; 2004.

79. Krzyzanowski M., Robbins D.R., Lebowitz M.D. Smoking cessation and changes in respiratory symptoms in two populations followed for 13 years. Int. J. Epidemiol. 1993; 22: 666-673.

80. Anthonisen N., Connet J., Kiley J. et al. Effects of smoking intervention and the use of an inhaled anticholinergic bronchodilator on the rate of decline of $\mathrm{FEV}_{1}$ : the Lung Health Study. J. A. M. A. 1994; 272: 1497-1505.

81. Scanlon P.D., Connett J.E., Waller L.A. et al. Smoking cessation and lung function in mild-to-moderate chronic obstructive pulmonary disease. The Lung Health Study. Am. J. Respir. Crit. Care Med. 2000; 161: 381-390.

82. Scharf D., Shiffman S. Are there gender differences in smoking cessation, with and without bupropion? Pooled- and meta-analysis of clinical trials of bupropion SR. Addiction. 2004; 99: 1462-1469.

83. Gritz E.R., Nielsen I.R., Brooks L.A. Smoking cessation and gender: the influence of physiological, psychological, and behavioral factors. J. Am. Med. Wom. Assoc. 1996; 51: 35-42.

84. Bjornson W., Rand C., Connett J.E. et al. Gender differences in smoking cessation after 3 years in the Lung Health Study. Am. J. Publ. Hlth 1995; 85: 223-230.

85. Panday S., Reddy S.P., Ruiter R. et al. Nicotine dependence and withdrawal symptoms among occasional smokers. J. Adolesc. Hlth 2007; 40: 144-150.

86. O'Hara P., Connett J.E., Lee W.W. et al. Early and late weight gain following smoking cessation in the Lung Health Study. Am. J. Epidemiol. 1998; 148 (9): 821-830.

87. Wise R.A., Enright P.L., Connett J.E. et al. Effect of weight gain on pulmonary function after smoking cessation in the Lung Health Study. Am. J. Respir. Crit. Care Med. 1998; 157 (3): 866-872.

88. Dales R.E., Mehdizadeh A., Aaron S.D. et al. Sex differences in the clinical presentation and management of airflow obstruction. Eur. Respir. J. 2006; 28: 319-322.

89. Ленней Дж., Иннс Дж.А., Кромптон Дж.К. Неправильное использование ингаляторов: оценка ингаляционной техники и предпочтений пациентов при сравнении семи различных ингаляционных устройств. Пульмонология 2005; 4: 80-84.

90. Goodman D.E., Israel E., Rosenberg M. et al. The influence of age, diagnosis and gender on proper use of metered dose inhalers. Am. J. Respir. Crit. Care Med. 1994; 150: 1256-1261.

91. Vestbo J., Soriano J.B., Anderson J.A. et al. Gender does not influence the response to the combination of salmeterol and fluticasone proprionate in COPD. Respir. Med. 2004; 98: $1045-1050$.

92. O'Donnell D.E., Fluge T., Gerken F. et al. Effects of tiotropium on lung hyperinflation, dyspnoea and exercise tolerance in COPD. Eur. Respir. J. 2004; 23: 832-840.

93. Convery R.P., Leitch D.N., Bromly C. et al. Effect of inhaled fluticasone propionate on airway responsiveness in treatment-naive individuals-a lesser benefit in females. Eur. Respir. J. 2000; 15: 19-24.

94. Decramer M., Celli B., Tashkin D.P. et al. Clinical trial design considerations in assessing long-term functional impacts of tiotropium in COPD: The UPLIFT trial. COPD 2004; 1: 303-312.

95. Tashkin D.P., Celli B., Senn S. et al. for the UPLIFT Study Investigators. A 4-year trial of tiotropium in chronic obstructive pulmonary disease. N. Engl. J. Med. 2008; 359: 1543-1554.

96. Cooper J.D., Trulock E.P., Triantafillou A.N. et al. Bilateral pneumonectomy (volume reduction) for chronic obstructive pulmonary disease. J. Thorac. Cardiovasc. Surg. 1995; 109: 106-119.

97. National Emphysema Treatment Trial Research Group. Patients at high risk of death after lung volume reduction surgery. N. Engl. J. Med. 2001; 345: 1075-1082.

98. Haggerty M.C., Stockdale-Woolley R., Zuwallack $R$. Functional status in pulmonary rehabilitation participants. J. Cardiopulm. Rehabil. 1999; 19: 35-42.

99. Foy C.G., Rejeski W.J., Berry M.J. et al. Gender moderates the effects of exercise therapy on health-related quality of life among COPD patients. Chest 2001; 119: 70-76.

\section{Информация об авторах}

Овчаренко Светлана Ивановна - д. м. н., проф. кафедры факультетской терапии № 1 лечебного факультета ГОУ ВПО "ММА им. И.М.Сеченова"; тел. / факс: (499) 248-56-67; e-mail: svetftk@mail.ru

Капустина Валентина Андреевна - аспирант кафедры факультетской терапии № 1 лечебного факультета ГОУ ВПО "ММА им. И.М. Сеченова"; тел.: (499) 248-45-23; e-mail: kapustina@pochta.ru 